\title{
Las tecnologías de la información y comunicaciones (TIC) y la brecha digital: su impacto en la sociedad del conocimiento del Perú
}

\author{
CPC Carlos Pastor Carrasco
}

Docente Auxiliar de la Facultad de Ciencias Contables

\author{
- Ahora digo — dijo a esta sazón Don Quijote- \\ que el que lee mucho y anda mucho, \\ ve mucho y sabe mucho
}

\section{RESUMEN:}

El objetivo de la sociedad del conocimiento es alcanzar que las sociedades del conocimiento sean fuentes de desarrollo para todos, y sobre todo para los paises menos adelantados. El propósito del presente artículo es analizar el papel de la brecha digital en las sociedades del conocimiento como causales de la exclusión de las empresas e individuos en el uso de las tecnologías de la información y comunicación (TIC) en el Perú.

Podemos decir que hoy nos encontramos ante una nueva forma de exclusión, la denominada «brecha digital", que es capaz de ampliar el abismo que separa a las regiones y a los paises (brecha digital internacional) e inclusive a los grupos de ciudadanos de una sociedad (brecha digital doméstica). La brecha cognitiva pone de manifiesto el potencial de exclusión que pueden conllevar las sociedades del conocimiento, cuando su desarrollo se limita a promover una economía del conocimiento.

Asimismo, el trabajo aporta cifras de la brecha digital en Perú obtenidas en la Oficina Nacional de Gobierno Electrónico e Informática (ONGEI) durante los años 2000 al 2008, tanto en los hogares como en las empresas, a fin de poner de manifiesto que existe un acceso desigual entre las zonas geográficas del país, que no solo depende de la infraestructura disponible, sino de las habilidades de la población para utilizar las tecnologías de la información y comunicación (TIC).

Palabras clave: Brecha digital, sociedad de la información, sociedad del conocimiento, tecnologías de información y comunicación (TIC). 


\section{INTRODUCCIÓN}

La expresión «sociedad del conocimiento» fue utilizada por primera vez en 1969 por Peter Drucker $^{1}$, y en el decenio de 1990 fue profundizada en una serie de estudios detallados publicados por investigadores como Nico Ste$\mathrm{hr}^{2}$, o Frank Webster ${ }^{3}$. Quienes la concibieron estimaban que, en el futuro, la riqueza de una nación iba a depender más de su capacidad para producir, intercambiar y transformar conocimientos, que de sus riquezas naturales o su producción manufacturada.

¿Podremos decir que el siglo XXI será la era de las sociedades del aprovechamiento compartido? Son muchos los factores que suponen un obstáculo para el cumplimiento de dicho objetivo. Por ejemplo, el concepto de «brecha digital» que, a menudo, va acompañada por una «fractura cognitiva». Esta última crea una divisoria entre los países que poseen sistemas eficaces de educación, investigación e innovación, por un lado, y las naciones que se ven azotadas por el flagelo de la fuga de cerebros, por otro lado.

En el Informe mundial de la UNESCO titulado "Hacia las sociedades del conocimiento", se presenta un panorama prospectivo de los cambios radicales ocurridos en nuestros días y se destacan, entre otras, las siguientes ideas clave:

- Las sociedades del conocimiento no son meras sociedades de la información. En efecto, a diferencia de la información, el conocimiento no se puede considerar una mercancía cualquiera.
- La reducción de la «brecha digital» es una tarea importante, pero no es suficiente de por sí, ya que esa brecha va acompañada con mucha frecuencia por una «brecha cognitiva» mucho más honda y antigua.

- El conocimiento va a desempeñar un papel cada vez más importante en el desarrollo económico, tanto en los países del hemisferio norte como en los del hemisferio sur. Asimismo, el conocimiento es un factor clave del desarrollo humano sostenible.

- El auge de las sociedades del conocimiento ofrece una nueva posibilidad de desarrollo a los países del hemisferio sur, con tal de que no desemboque en la creación de rentas de situación en beneficio de los países del hemisferio norte.

- No hay un modelo único de sociedad del conocimiento. Las sociedades del conocimiento han de ser pluralistas y deben reconocer la diversidad de las culturas cognitivas. A cada sociedad le corresponde valorizar los conocimientos locales y autóctonos que posee.

- Las sociedades del conocimiento sólo cumplirán su misión si saben cultivar una ética de la colaboración y si aciertan a convertirse en sociedades donde el saber sea objeto de un aprovechamiento compartido.

Por otro lado, es necesario otorgar prioridad a la diversidad lingüística, estimulando el bilingüismo desde la enseñanza primaria $y$, en la medida de lo posible, lograr el trilingüismo, lo que nos servirá para avanzar

1 En 1974, Peter Drucker escribió su libro La sociedad post-capitalista, en el que destacaba la necesidad de generar una teoría económica que colocara al conocimiento en el centro de la producción de riqueza. Al mismo tiempo, señalaba que lo más importante no era la cantidad de conocimiento, sino su productividad. En este sentido, reclamaba para una futura sociedad, para una sociedad de la información en la que el recurso básico sería el saber, que la voluntad de aplicar conocimiento para generar más conocimiento debía basarse en un elevado esfuerzo de sistematización y organización.

2 Knowledge societies, Nico STEHr, 1994, London: Thousand Oaks.

3 Theories of the Information Society, Frank WeBster, 2002, Routledge. 
en obtener el conocimiento en el mundo. Asimismo, conviene promover la diversidad lingüística en el ciberespacio, apoyándose en dispositivos adecuados.

Es menester avanzar hacia una certificación de los conocimientos en Internet, esto es, hacia el establecimiento de homologaciones de la calidad que permitan identificar los sitios donde la información es especialmente fiable y valiosa.

\section{UNA NUEVA REVOLUCIÓN TECNOLÓGICA}

Ha transcurrido poco más de un siglo de la última revolución industrial, por ello el mundo y nuestro país asisten a una nueva revolución tecnológica, cuyo impacto superará ampliamente al que trajo consigo la popularización de la imprenta y el libro.

La CEPAL sostiene que la brecha digital tiene dos dimensiones: extensión (acceso) y profundidad (calidad de acceso). Aunque se llegue a una situación en la que todos los habitantes tengan acceso a redes digitales, seguramente algunos tendrán acceso a un ancho de banda cada vez mayor y podrán usar servicios multimedia avanzados, mientras que otros estarán limitados a las comunicaciones de voz. En este contexto surgen dos desafíos: la eficiencia, para asegurar la calidad de los servicios al menor precio posible, y la equidad, para posibilitar un adecuado proceso de difusión.

Esta revolución tecnológica resulta de la convergencia de diversos fenómenos, entre los que destacan la difusión mundial de redes de información y comunicaciones (como internet), la informatización de bienes y procesos, la digitalización de la información y la creciente importancia del aspecto inmaterial de la riqueza producida. Emerge un nuevo paradigma económico y social caracterizado por la confluencia de cinco procesos:

- La integración digital de sonido, datos a imagen, así como la convergencia entre telecomunicaciones, computación y televisión. De esta forma, las distinciones tradicionales entre telefonía, televisión, ondas radiales y TV cable tienden a desaparecer. Se tornará cada vez más irrelevante la diferencia entre medios de acceso (computador, televisión y otros artefactos) a las redes digitales de información, mientras estas ofrecerán nuevos servicios cada vez más interactivos.

- La difusión cada vez más genérica de las nuevas tecnologías de información y comunicación. Sus usos tenderán a extenderse a todos los ámbitos de la vida económica y social, se combinarán con otras tecnologías y facilitarán los procesos de cambio organizacional de comunidades, empresas a instituciones.

- La maximización de la eficiencia y eficacia social de las nuevas tecnologías cuando operan en redes. Es decir, no solo se trata de redes físicas con computadores y cables de fibra óptica, sino redes sociales y comerciales de información y conocimiento, que aumentarán su utilidad para los usuarios y para la sociedad en la medida que más personas y empresas estén conectadas a ellas.

- La aceleración de la producción y difusión global del conocimiento y la información. Las nuevas tecnologías potencian la sinergia entre conocimiento e innovación. Esto implica que mientras más invierta el país en recursos humanos, mayor será su dominio sobre estas nuevas tecnologías y mayor será su capacidad de innovar, generando nuevas bases de competitividad y bienestar social.

- El desarrollo de una nueva infraestructura de información. Su rol será tan vital para 
el crecimiento y el bienestar como actualmente lo es la infraestructura física. La multiplicación de las redes digitales, y el enriquecimiento del contenido que viaja por ellas, facilitará el desarrollo de empresas y mercados, el funcionamiento eficiente y descentralizado del sector público, así como el tránsito hacia una sociedad civil cada vez más abierta y comunicativa.

El desarrollo y difusión de cada uno de estos procesos está generando transformaciones que se deberán acelerar en la próxima década. En el ámbito económico, la automatización de la producción, la informatización de empresas y la difusión de mercados electrónicos se incrementará rápidamente. Surge, entonces, el desafío de construir nuevas ventajas competitivas. Para lograrlo, es necesario aumentar la incorporación de nuevos conocimientos a innovaciones a la producción de bienes y servicios, formar una fuerza de trabajo de calidad mundial y estimular la emergencia de nuevos sectores intensivos en tecnologías digitales, tales como servicios productivos, industria del turismo, servicios financieros, servicios de educación, así como las industrias culturales y audiovisuales, entre otros.

Nuestra sociedad experimentará cambios sustantivos en su vida cotidiana, puesto que un verdadero arsenal de innovaciones transformará radicalmente sus formas de trabajo y estudio, sus hábitos de cultura y entretenimiento, el modo como comercian, se informan o comunican, y la manera como acceden a servicios básicos como el transporte, la educación y la salud. Acelerar la masificación y la universalización del acceso a los beneficios que conllevan estas nuevas tecnologías adquiere, entonces, una importancia estratégica.

El Estado, en tanto, productor de servicios e información por excelencia, sufrirá importantes mutaciones en la medida que siga incrementando su informatización y el uso de las redes electrónicas. El desafío en este ámbito consiste en acelerar el cambio institucional para maximizar el uso eficiente de las nuevas tecnologías. Así, se podrá modernizar la gestión pública y profundizar la descentralización del Estado, abriendo nuevos caminos para lograr el desarrollo sustentable, el bienestar social y la expansión de una sociedad civil más abierta, participativa y ciudadana.

\section{¿HAY QUE REPENSAR LA FORMACIÓN PROFESIONAL EN LA SOCIEDAD DEL CONOCIMIENTO?}

El desarrollo de la sociedad del conocimiento en la que la gestión de la información y de la innovación constituye elemento clave del proceso de cambio, exige, sin lugar a dudas, una población mayoritariamente formada. Si el éxito de la industrialización en sus primeras etapas requirió una población alfabetizada, si luego, el desarrollo de la automatización exigió una población con una educación básica, la sociedad del conocimiento requiere una población con una educación secundaria completa.

Ahora bien, a qué tipo de formación nos referimos. ¿Es un problema de cantidad? ¿Es suficiente con generalizar la educación secundaria a toda persona que se incorpore al mercado de trabajo? Los mecanismos cognitivos de la sociedad del conocimiento requieren dos tipos de premisas básicas que deberían cumplir todos los sistemas de formación:

- Una fuerte capacidad de aprendizaje sobre la que basar la innovación y la capacidad de adaptación rápida a los cambios.

- La actualización permanente de los conocimientos. 
Hasta ahora, la capacidad de aprendizaje iba relacionada con los niveles de educación inicial. A mayor educación, los individuos suelen demostrar mayor capacidad de aprender. Con lo cual, comparativamente, la educación general tiene mayor ventaja en relación con la formación especializada que dedica más esfuerzos al dominio de técnicas y aptitudes específicas que en incrementar las capacidades genéricas de aprender de los individuos. Por esta razón la formación profesional ha ido evolucionando históricamente desde una concepción utilitarista ocupacional basada en el aprendizaje de las aptitudes básicas de los oficios hacia una formación más basada en los "porqués» del oficio. De ahí que, muy a menudo, al finalizar una formación profesional, aún sea necesario un período de entrenamiento para conseguir un trabajador operativo.

Dentro de esos dos límites se ha ido manteniendo el equilibrio de los contenidos de la formación profesional. Sin embargo, la evolución de los requerimientos profesionales en el contexto de la sociedad del conocimiento está cuestionando dicho equilibrio: El intenso proceso de automatización de procesos, la importancia que adquieren en las tareas, incluso técnicas, la gestión de volúmenes cada vez más importantes de información, y la complejidad creciente de la toma de decisiones, exige otro perfil de cualificación en el que la relación entre "comprensión» y «ejecución» se balancea a favor de la primera.

En ese contexto, convendría replantear la distancia que actualmente separa una formación profesional dedicada cada vez con mayor intensidad a proveer capacidades de comprensión especializadas y la educación general. Habría que vislumbrar una mayor integración entre las dos ramas tradicionales de la educación secundaria, básicamente dedicada a desarrollar las capacidades de comprensión especializadas que debería ser complementada con una formación posterior, pero ya en las primeras fases de la incorporación al mundo laboral, en un marco de actualización permanente de los conocimientos y de adaptación a los cambios y a las innovaciones. Es decir, ya en el contexto de la formación continua. La clásica división, y motivo de grandes polémicas en el pasado, entre la formación cultural general y la tecnológica profesional ya no tiene sentido. Una de las características de la sociedad del conocimiento es que su base tecnológica afecta tanto el mundo profesional como el personal y social.

La mayor capacidad de resolución de problemas se adquiere con la experiencia y con un mayor nivel de cualificación, pero la experiencia, con la velocidad de cambio a la que está sometida la sociedad del conocimiento, escasea por definición. No hay tiempo para adquirirla. Hoy se constata que para ejercer ciertos puestos de trabajo se requieren unas competencias que normalmente se asocian a titulaciones medias o superiores. Pero los resultados no son satisfactorios porque el problema no es de más nivel de educación, y eso ocurre tanto para un albańil como para una secretaria, para un director de establecimiento comercial o un gerente.

La Unión Internacional de Telecomunicaciones realizó en el año 2003 el Índice de acceso digital de la UIT: la primera clasificación completa de las TIC en el mundo, llegando a la conclusión de que la educación y la asequibilidad son factores clave para impulsar la adopción de nuevas tecnologías.

Los países se clasifican en una de las cuatro siguientes categorías de acceso digital: elevado, medio alto, medio bajo y bajo. En la categoría media alta figuran principalmente naciones de Europa Central y Oriental, el Caribe, los Estados árabes y los países latinoamericanos con economías emergentes. 
Muchos de estos países han utilizado las TIC como motor del desarrollo y sus políticas gubernamentales los han ayudado a llegar a un nivel impresionante de acceso TIC. En este contexto, hay que citar destacadamente importantes proyectos TIC como la Ciudad Internet de Dubai en los Emiratos Árabes Unidos (país árabe mejor puntuado por el IAD), el Supercorredor Multimedios de Malasia (nación asiática con la mayor puntuación) y la Ciudad Cibernética de Mauricio, que junto con Seychelles, fue el país mejor puntuado en África. El IAD será una herramienta muy útil para seguir los progresos que se realicen en el futuro en estos ambiciosos países con economías incipientes.

El mayor avance registrado en cuanto a las TIC durante los últimos cuatro ańos corresponde a los cuatro tigres asiáticos y a Japón. Este avance sugiere que el inglés ya no es un factor decisivo en cuanto a la rápida adopción de tecnología, ya que la mayoría del contenido se encuentra disponible en otros idiomas.

\section{SITUACIÓN ACTUAL DE LA PENETRACIÓN DE LAS TIC EN PERÚ}

Durante el año 2003, en la Cumbre Mundial sobre la Sociedad de la Información en Ginebra, los líderes mundiales declararon en su punto 10: «Somos plenamente conscientes de que las ventajas de la revolución de la tecnología de la información están en la actualidad desigualmente distribuidas entre los países desarrollados y en desarrollo, así como dentro de las sociedades. Estamos plenamente comprometidos a convertir la brecha digital en una oportunidad digital para todos, especialmente aquellos que corren peligro de quedar rezagados y aún más marginados» ${ }^{4}$.

Las TIC no operan en el vacío, si no están presentes las condiciones precursoras para el aprovechamiento integral entre las TIC y la competitividad, es probable que se presente una "paradoja de productividad», es decir, que si las inversiones en TIC no van acompañadas de los esfuerzos complementarios que favorecen sus beneficios, los frutos de estas inversiones pueden ser escasos.

El problema de acceso a TIC no solo implica una brecha digital entre el Perú y otros países, sino un rezago digital al interior del país, marcado por una desigualdad en el desarrollo de TIC en la sociedad. La evidencia indica que las brechas digitales se deben a diferencias culturales, de edad e ingresos, entre otros.

La calificación obtenida en el Índice de acceso digital de la UIT, le dio el puntaje de 0.44 como de acceso medio bajo, Chile fue calificada como 0.58, EE.UU. 0.78 y Suecia 0.85 , el más alto índice obtenido.

Cerca del 40\% de los ciudadanos de Perú respondieron a una encuesta señalando que no contaban con un computador o no podían permitirse utilizar servicios internet, lo cual apunta a que la asequibilidad es un factor indispensable de éxito. La tenencia de computadoras en el hogar se mantiene baja en los hogares del Perú. Así tenemos que en el año 2000, el 4,4\% de los hogares tenían computadoras, en el período 2003/2004 la ENAHO mostró que este porcentaje se incrementó a 6,8\%. Para el trimestre evaluado enero-febrero-marzo 2008 el porcentaje se incrementó a $15,5 \%$.

Al considerar el área de residencia, encontramos importantes diferencias en hogares con tenencia de computadoras; dife-

4 http://www.itu.int/wsis/documents/doc_multi.asp?lang=es\&id=1161|0 
rencias que se han mostrado también en la disponibilidad de servicios TIC.

Para el trimestre evaluado, el 26,6\% de los hogares de Lima Metropolitana dispone de una computadora, $20,7 \%$ de los hogares del resto urbano y $1,2 \%$ de los hogares rurales.

Estos porcentajes muestran diferencias significativas en los resultados obtenidos en similar trimestre del año anterior, sobre todo en Lima Metropolitana.

\section{Hogares con computadora según uso}

La última información de ENAHO muestra que en el 94,4\% de los hogares que disponen de computadora, esta es usada para el hogar, es decir, para actividades académicas, profesionales, estudio; $1,4 \%$ de hogares usa la computadora para propósitos de trabajo y, 4,2\% combina su uso en el hogar y trabajo. Los porcentajes obtenidos en similar trimestre del año anterior, 95,2\% lo usa para el hogar, $0,6 \%$ para el trabajo y $4,2 \%$ para el hogar y el trabajo, no distan mucho de los registros del trimestre enero-febrero-marzo 2008.

\section{Tenencia de TIC y presencia de nińos menores de 16 años en el hogar}

Sin embargo, la situación es muy diferente cuando se analizan los mismos indicadores por rango de edad. La presencia de nińos en un hogar aparece como un factor importante en el acceso a las TIC y, particularmente, internet; para el trimestre enero-febrero-marzo 2008, 57,6\% es la proporción de hogares en los cuales hay presencia de niños menores de 16 años y que disponen de una conexión a Internet dentro del hogar.

La proporción de hogares que tienen una computadora y su relación con la presencia de nińos menores de 16 ańos en el hogar, muestra para el trimestre evaluado un $68,1 \%$ de hogares que disponen de una computa- dora y que a su vez hay presencia de niños menores de 16 ańos en el hogar.

La mayor penetración, tanto de computadoras como de internet, se observa entre peruanos de 19 a 49 años, grupo compuesto por estudiantes, profesionales y trabajadores que utilizan TIC en sus actividades. «En el otro extremo se ubica el grupo más rezagado en el uso de las tecnologías, los adultos de más de 50 años».

\section{Pirámide de internautas, según grupos de edad}

La brecha digital es también principalmente un problema de edad y educación, donde el problema de la diferencia de género es muy pequeño. Así lo muestra la pirámide de internautas que corresponde al trimestre actual, donde la proporción de usuarios de internet con edades entre los 6 a 18 ańos tiene una gran diferencia con las personas de 41 y más años de edad que accedan a internet en cualquiera de los lugares como: el hogar, trabajo, establecimiento educativo, cabina pública u otro lugar.

Asimismo, se observa que el grupo de edad con mayor acceso a internet es el de 12 a 18 años; en hombres el porcentaje de internautas alcanza el 27,3\%; mientras que el de mujeres $31,5 \%$. Igualmente a medida que avanza la edad, hay un decrecimiento en el acceso a internet.

El bajo acceso se explica por la combinación de factores, como son una falta de capacitación en el uso de tecnologías, el rechazo cultural a estas herramientas y la carencia de beneficios específicos. Si bien la edad explica algunos de los rezagos en la adopción de TIC, el nivel socioeconómico, relacionado con el estilo de vida y los ingresos mensuales, es el mayor determinante de la brecha digital. La brecha digital interna en el Perú es enorme, la población de menores ingresos 
que utilizan TIC está muy por debajo de la media poblacional.

\section{Tenencia de computadoras de las empresas por sectores económicos}

La brecha digital en Perú no solo afecta a la población en general. De hecho, la razón por la cual es tan grande la brecha entre las personas es que no tienen incentivos para utilizar la tecnología, puesto que ni siquiera en su lugar de trabajo las TIC son parte de las herramientas que se utilizan cotidianamente.

La adopción de TIC en las empresas peruanas es tan heterogénea como las desigualdades económicas que hay entre las empresas y la educación de los empleados. La situación es más crítica aun en las micro y pequeñas empresas. En consecuencia, el porcentaje de trabajadores con acceso a computadoras y a internet también está fuertemente sesgado.

\section{CUADRO 2. COMPUTADORAS POR SECTORES}

\begin{tabular}{|l|c|c|}
\hline $\begin{array}{l}\text { TENENCIA DE } \\
\text { COMPUTADORAS }\end{array}$ & PRODUCCIÓN & SERVICIOS \\
\hline Tienen & $76.3 \%$ & $82.8 \%$ \\
\hline No tienen & $23.7 \%$ & $17.2 \%$ \\
\hline Total & $100 \%$ & $100 \%$ \\
\hline
\end{tabular}

FuENTE: INEI - Concytec - Encuesta de Ciencia, Tecnología e Innovación Tecnológica 1999.

Haciendo un análisis sectorial observamos que el $76.3 \%$ de las empresas dedicadas a la producción de bienes tienen o cuentan con computadoras y el $23.7 \%$ de las restantes no tienen; por otro lado, de las empresas que están orientadas a los servicios, el $82.8 \%$ tiene o cuenta con computadoras y el $17.2 \%$ no tiene.

Estas cifras nos hacen pensar que las empresas dedicadas a los servicios son las que están empleando e implementando tecnología informática más que sus similares en el sector de producción.
Y esta tendencia del uso de computadoras por las empresas de servicios es bastante realista, en la medida que las empresas de este sector son las que a la fecha utilizan más tecnología digital en sus trabajos. En tanto que, en el sector de producción encontramos que aún existen muchas empresas que no han modernizado sus procesos productivos y menos aún incorporado en las más importantes áreas, como en administración, sistemas computarizados para la gestión empresarial.

Entre los problemas relacionados con la baja penetración de las TIC en las empresas, destacan: la regulación del sector de telecomunicaciones, las tarifas que se cobran por el uso de la banda ancha, los tamaños desiguales de las empresas y la falta de financiamiento para adquirir equipos de cómputo. En este sentido, los mismos síntomas que muestran los hogares, surgen en las empresas. Por ello, hay una profunda brecha digital de TIC en las empresas peruanas comparadas con otros países. Además, tal brecha es variable y depende del tamańo de la empresa, de los sectores económicos en donde operan las empresas y de las zonas geográficas del país.

\section{CONCLUSIONES}

1. En general, las empresas en el país no cuentan con la infraestructura de cómputo adecuada.

2. Las empresas del sector de servicios son las que más innovaciones de tecnologías están absorbiendo que las empresas del sector productivo.

3. El nivel de inversiones en el país en modernización de tecnologías es muy bajo, y tal vez, la razón fundamental es el que el país aún se encuentra dentro del pro- 
ceso de recesión económica con lo que se espera que las inversiones se estén postergando a la espera de mejores situaciones económicas.

4. El número de trabajadores que usan computadoras para el desarrollo de sus actividades dentro de la empresa es también relativamente bajo.

5. El número de trabajadores que tienen acceso a internet es otro de los datos que se encuentra en los niveles bajos.

6. Poder acceder a las tecnologías de la información y comunicaciones (TIC) es un requisito importante para participar de una sociedad cada vez más dependiente de la tecnología. Las TIC serán un elemento dinamizador fundamental en la sociedad. Por consiguiente, quienes, individual y colectivamente, logren desarrollar la infraestructura y las capacidades para utilizarlas serán privilegiados, tendrán mayor capacidad de decisión e influirán en la construcción de la sociedad del conocimiento.

7. El conocimiento permite diseñar, producir y exportar tecnologías, es decir, la producción intelectual en áreas disciplinarias específicas en las sociedades del conocimiento está orientada a satisfacer necesidades propias de cada país. En muchos casos, estos avances benefician también a otros países, pues a través de la importación de tecnologías, métodos y herramientas, mejoran su desarrollo científico y tecnológico.

8. La forma más rápida y efectiva de revertir la tendencia negativa de la competitividad del país es mejorar la eficiencia en el uso de los factores de producción. Para ello, uno de los caminos más eficaces es la adopción de tecnologías de información y comunicaciones (TIC). Dada la pérdida de competitividad que experimenta el país y la forma como las TIC contribuyen a revertir dicha tendencia, es prioritario que Perú plantee una visión a largo plazo para la adopción de TIC. En este sentido, el primer paso es entender la situación actual en el uso y aprovechamiento de las TIC y las acciones para promover el uso de dichas tecnologías.

\section{REFERENCIAS}

Angulo, Carlos; Toro, José (2001). «La universidad 'académicamente abierta' para la actual sociedad del conocimiento». En: L. E. Orozco (comp.). Educación superior en el siglo XXI: desafío global y respuesta nacional. Bogotá: Universidad de los Andes.

BARnett, Ronald (2001). Los límites de la competencia. El conocimiento, la educación superior y la sociedad. Barcelona: Gedisa.

Cabrera, José (2004). «Navigators and castaways in cyberspace: psychosocial experience and cultural practices in school children's appropriation of the Internet». En: M. Bonilla; G. Cliché (eds.). Internet and Society in Latin America and the Caribbean (pp. 2186). [Versión electrónica]. Ontario: Southbound / IDRC Books. Fecha de consulta: 02/07/08.

http://www.idrc.ca/en/ev-84512-2011-DO_TOPIC.html

Cepal (2003a). «Declaración de Bávaro». En: Conferencia Ministerial Regional Preparatoria de América Latina y el Caribe para la Cumbre Mundial sobre 
la Sociedad de la Información. (2931 de enero de 2003: Bávaro, Punta Cana, República Dominicana)». [Versión electrónica]. Fecha de consulta: 30/06/08.

http://www.eclac.cl/prensa/noticias/ noticias/9/11719/Bavarofinalesp.pdf

Cepal (2008). «La sociedad de la información en América Latina y el Caribe: desarrollo de las tecnologías y tecnologías para el desarrollo». [Versión electrónica]. Fecha de consulta: 30/06/08.

http:/www.eclac.cl/socinfo/noticias/ noticias/1/32291/2007-1081-TICsSociedad_informacion-FINAL.pdf

Cepal (2008). «América Latina y el Caribe en la transición hacia una sociedad del conocimiento. Una agenda de políticas públicas». [Versión electrónica]. Fecha de consulta: 30/06/08.

http://www.eclac.cl/publicaciones/ $\mathrm{xml} / 2 / 4312 / \mathrm{lcl} 1383 \mathrm{e} . \mathrm{pdf}$

CMSI (Cumbre Mundial para la Sociedad de la Información) (2003). «Declaración de Principios y Plan de Acción», Ginebra. [Versión electrónica]. Recuperada el 25 de junio de 2008.

http://www.itu.int/wsis/ d o cuments/doc_multi. asp?lang=en \&id $=1161 \mid 1160$
Cornella, Alfons (2000). La gestión de la información en la organización. Bilbao: Deusto.

ITU (Unión Internacional de Telecomunicaciones) (2003). «Digital Access Index: World's First Global ICT Ranking. Education and Affordability Key to Boosting New Technology Adoption». Ginebra. [Versión electrónica]. Fecha de consulta: 01/06/08.

http://www.itu.int/newsarchive/ press_releases/2003/30.html

Mettlen, Peter (Primavera 2005). «The coming global knowledge society: how to analyze and shape its future?». Futures Research Quarterly. Vol. 21, n. ${ }^{\circ}$ 1, pp. 51-68.

ONGEI. «Indicadores de Tecnologías de Información y Comunicación en las Empresas». Lima, Nov 2001. [Versión electrónica]. Recuperada el 24 de junio de 2008. http://www.ongei.gob.pe/publica/indicadores/Lib5136/Libro.pdf

ONGEI. «Las Tecnologías de Información y Comunicación en los Hogares Lima», Jun 2008. [Versión electrónica]. Recuperada el 26 de junio de 2008. http://www.ongei.gob.pe/publica/indicadores/hogares/TICH-ENE2008MAR2008.pdf 